\title{
Dark matter searches with the ANTARES neutrino telescope: constraints to CMSSM and mUED models
}

\author{
Juan de Dios Zornoza*i \\ IFIC, Instituto de Física Corpuscular (CSIC - Univ. de Valencia, Ed. Institutos de Investigación, \\ AC 22085, E-46071 Valencia, Spain \\ E-mail: zornozalific.uv.es
}

Guillaume Lambard

IFIC, Instituto de Física Corpuscular (CSIC - Univ. de Valencia, Ed. Institutos de Investigación), AC 22085, E-46071 Valencia, Spain

E-mail: lambardeific.uv.es

\begin{abstract}
ANTARES is the largest neutrino telescope Northern hemisphere. It consists of a threedimensional array of 885 photomultipliers to collect the Cherenkov light induced by relativistic muons produced in $\mathrm{CC}$ interactions of high energy neutrinos. One of the main scientific goals of the experiment is the search for dark matter. We present here the analysis of the recently unblinded data taken during 2007 and 2008 to look for a WIMP signal in the Sun. WIMPs are one of the most popular scenarios to explain the dark matter content of the Universe. They would accumulate in massive objects like the Sun or the Galactic Center and their self-annihilation would produce (directly or indirectly) high energy neutrinos detectable by neutrino telescopes. Contrary to other indirect searches (like with gamma rays or positrons), the search for neutrinos in the Sun is free from other astrophysical contributions, so the explanation of a potential signal in terms of dark matter would be much more robust. The results are interpreted within two theoretical frameworks: CMSSM and mUED.
\end{abstract}

36th International Conference on High Energy Physics,

July 4-11, 2012

Melbourne, Australia

${ }^{*}$ Speaker.

${ }^{\dagger}$ On behalf of the ANTARES collaboration 


\section{Introduction}

There is a lot of experimental evidence about the existence of dark matter in the Universe, including the first hints by Zwicky in 1933 [1], the observations from WMAP [2], the results on the Big Bang Nucleosynthesis [3], the rotation curves of galaxies [4] and the studies of highly red-shifted Ia supernovae [5]. From these results we can conclude that only $27 \%$ of the Universe content is matter (the rest being dark energy) and that $80 \%$ of the matter is non-baryonic. We do not know the nature of this non-baryonic component. What we do know is that it cannot be dominated by relativistic particles like neutrinos, since this would be incompatible with the largescale structure of the Universe. We have to look outside the Standard Model. There are several conditions that these new particles should fulfill: to have an interaction cross-section of the order of that for weak interactions (so that they can provide the infered relic density), to be massive and to be stable. We often refer to a generic kind of particles fulfilling these conditions as WIMPs (Weaking Interacting Massive Particles). In the analysis presented here, we will focus on two frameworks where WIMPs can be accomodated: SuperSymmetry (SUSY) and Universal Extra Dimensions (UED). In particular, we have considered two constrained versions: CMSSM [6] and mUED [7]. The corresponding particles are the neutralino and the lightest Kaluza-Klein particle, whose stability is guaranteed by the conservation of the R-parity and the KK-parity, respectively.

In this paper we will present the results of a binned search in the Sun direction with 20072008 data of the ANTARES neutrino telescope. Neutrino telescopes can contribute to the search for dark matter. Among the most interesting sources for these detectors we can mention the Sun, the Galactic Center and the Earth. WIMPs acumulate in the Sun after elastically scattering and become gravitationally trapped. If they are Majorana particles, they would self-annihilate and produce secondaries (in general, not directly neutrinos, but is some models, this channel is also possible). Then, these secondaries decay yielding neutrinos of energies that could be detectable in neutrino telescopes. One of the advantages of this kind of analysis, compared to searches in the Galactic Center (including those of gamma rays) or to cosmic ray experiments is that a potential signal would be very clean, since there are no other well motivated astrophysical explanations for high energy neutrinos from the Sun (the background produced by cosmic rays interacting in the Sun's corona is very small).

The structure of this paper is as follows. In Section 2, the ANTARES detector is described. Sections 3 and 4 explain the ingredients of the analysis (data and simulation) and the optimization procedure, respectively. The results are presented in Section 5. Finally, the conclusions are summarized in Section 6.

\section{The ANTARES neutrino telescope}

The ANTARES collaboration completed the construction of the first deep-sea neutrino telescope in 2008 [8]. It is located at $\left(42^{\circ} 48^{\prime} \mathrm{N}, 6^{\circ} 10^{\prime} \mathrm{E}\right)$ at a depth of $2475 \mathrm{~m}$ in the Mediterranean Sea, $42 \mathrm{~km}$ off-shore Toulon, France and consists of a three-dimensional array of photomultipliers (PMTs) which gather the Cherenkov light induced by relativistic muons produced by in the CC interactions of high-energy neutrinos with the matter in/around the detector. The total number of PMTs is 885 and they are installed along 12 vertical lines anchored to the sea bottom. The basic 

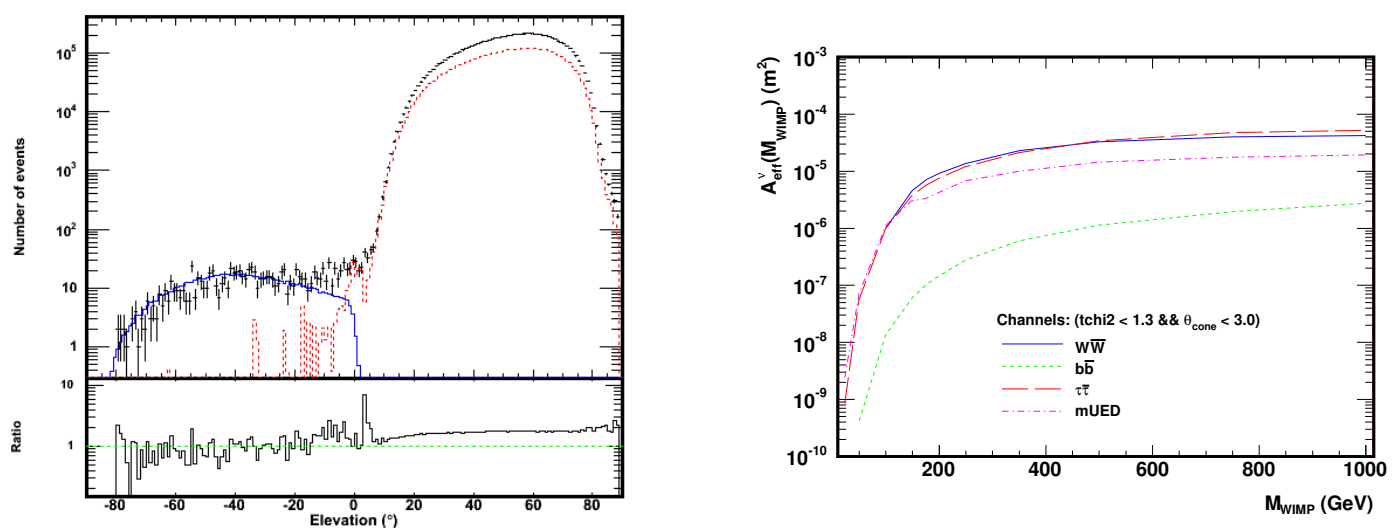

Figure 1: Left: Comparison between data and simulation: elevation of events. The distributions show the simulated atmospheric muons (red), the simulatd atmospheric upgoing-neutrinos (blue), and the reconstructed data (black). The ratio of data over simulation is shown below the corresponding plot. Right: Normalized effective area for different WIMP masses.

detector element is the Optical Module (OM), which consist of the 10-inch PMT and the glass sphere which protects it from the external pressure, together with the $\mu$-metal cage to attenuate the Earth's magnetic field and some special gel for a better optical coupling. The OMs are arranged in triplets and point 45 degrees downwards to avoid the effect of biofouling. The total length of the lines is $450 \mathrm{~m}$, although the lower $100 \mathrm{~m}$ do not contain OMs to avoid the effect of sea bottom dust. The separation between lines is 60-75 meters and the horizontal layout has an octogonal shape.

\section{Data and simulation}

As mentioned before, we have used for this analysis data of 2007-2008. Total number of active days is 375.3 , distributed in 185.5 in 2007, during which only 5 lines of the detector where conected, and 189.8 in 2008, when most or all the detector lines were taking data.

There are two kinds of background in the data. One the one hand, overhelmingly dominating, we have the atmospheric muons, produced by cosmic rays in the atmosphere. The way to reduce this background is two-fold. First, the detector is located at a large depth, so that the water above shields it against this background. Second, we select only up-going events, since atmospheric muons cannot traverse the Earth. Since the amount of atmospheric muons is very large, even with this selection we have down-going atmospheric muons which are mis-reconstructed as up-going. However, most of them can be removed by filtering out event with bad reconstruction quality, as explained in the next section.

The second kind of background are the atmospheric neutrinos produced after the interaction of cosmic rays in the atmosphere. This background cannot be avoided by only selecting up-going events, since they can traverse the Earth. In our analysis, we will use the direction information, since our signal is located around the Sun.

The amount of background is estimated from scrambled data, to reduce systematic uncertainties in the simulation (for instance, in the detector efficiency). In any case, data and simulation agrees well, as shown in Figure 1 (left). 
The signal is simulated with the WimpSim package [11], which allows to calculate the flux arriving at Earth for different annihilation channels. This allows to calculate the flux expected from a given model by weighting each channel accordingly. The two frameworks we have considered here are CMSSM and mUED. The main channels for our detector are $W^{+} W^{-}, b \bar{b}$ and $\tau \bar{\tau}$ for CMSSM and $c \bar{c}, b \bar{b}, \tau \bar{\tau}, t \bar{t}$ and $v \bar{v}$ for mUED. Once the flux at the detector is calculated, the actual signal is estimated by simulating with Monte Carlo the detector effective area.

\section{Cut optimization}

The final sample of events is chosen by optimizing the sensitivity. Until the final set of cuts are chosen, the data remains blinded. The parameters we use to make the optimization are a cut in the angular window around the Sun and a cut in the fit reconstruction quality $\chi^{2}$. This optimization is done separatedly for each channel and WIMP mass. The effective area obtained for $\chi_{t}^{2}<1.4$ and $\Psi<3^{\circ}$ (which a typical set of optimizing cuts) is shown in Fig. 1 (right).

\section{Results}

Once the data were unblinded, no significant excess was found, so flux upper limit have been set, as shown in Figure 2, for different models and WIMP masses. In CMSSM there is a large variation in the branching ratios for different parameter sets in the framework. For this reason we present the limits separatedly for the main channels $\left(W^{+} W^{-}, \tau \bar{\tau}, b \bar{b}\right)$. In the case of mUED, the spread among models is smaller, so the limit is shown for one particular set of parameters, which happens to be dominated by the $\tau \bar{\tau}$ channel.

Assuming equilibrium between capture and annihilation in the Sun, we can also set limits in the spin dependent cross section of WIMP-proton scattering [12], shown in Figure 3, compared with other experimental limits. It is also interesting to plot the available parameter space when several other experimental constrains (accelerators, WMAP, etc.) are included. This has been done with the package SuperBayess [13] for CMSSM and a modified version of its [14] for mUED.

\section{Conclusions}

We have presented the results of the search for WIMPs in the Sun using ANTARES data taken in the period 2007-2007. After data unblinding, no excess is found, so flux upper limits in the direction of the Sun have been set. Moreover, we have also obtained limits in the spin-dependent cross section for the WIMP proton scattering.

\section{Acknowledgements}

The author acknowledges the support of the Spanish MICINN Consolider-Ingenio 2010 Programme under grant MultiDark CSD2009-00064 and of the Prometeo Programme of the Generalitat Valenciana. 


\section{References}

[1] "Spectral displacement of extra galactic nebulae", F. Zwycky, Helv. Phys. Acta 6 (1933) 110-127.

[2] G. Hinshaw et al, WMAP Collaboration, "Five-Year Wilkinson Microwave Anisotropy Probe Observations: Data Processing, Sky Maps, and Basic Results", Astrophys. J. Supp. 180 (2009) 225âĂŞ245, arXiv:astro-ph/0803.0732

[3] K. Jedamzik and M. Pospelov, "Particle dark matter and Big Bang nucleosynthesis", Chapter 28 of "Particle Dark Matter: Observations, Models and Searches", Cambridge University Press, ISBN 9780521763684

[4] V. Rubin, W. K. Ford, Jr., "Rotation of the Andromeda Nebula from a Spectroscopic Survey of Emission Regions", Astrophysical Journal 159 (1970) 379.

[5] M. Kowalski et al., "Improved cosmological consraints from new, old and combined supernova data set", Astrophys. J. 686 (2008) 749-778, arXiv:astro-ph/0804.4142

[6] J. Ellis, K. Olive, "Supersymmetric dark matter candidates", chap. 8 of "Particle Dark Matter: Observations, Models and Searches", Cambridge University Press, ISBN 9780521763684

[7] H. C. Cheng, J. L. Feng and K. T. Matchev, "Kaluza-Klein dark matter", Phys. Rev. Lett. 89:211301 (2002), hep-ph/0207125

[8] M. Ageron et al., ANTARES collaboration, "ANTARES: The first undersea neutrino telescope", Nucl. Inst.. and Meth. in Phys. Res. A 656 (2011) 11-38, arXiv:1104.1607

[9] J.A. Aguilar et al., ANTARES collaboration, "Time Calibration of the ANTARES Neutrino Telescope", Astrop. Phys. 34 (2011) 539-549, arXiv:1012.2204

[10] J.A. Aguilar et al., ANTARES collaboration "A Fast Algorithm for Muon Track Reconstruction and its Application to the ANTARES Neutrino Telescope", Astropart. Phys. 34 (2011) 652-662, arXiv: arXiv:1105.4116

[11] J. Edsjö, "WimpSim Neutrino Monte Carlo", http://www.physto.se/edsjo/ wimpsim

[12] "Search for Dark Matter in the Sun with the ANTARES Neutrino Telescope in the CMSSM and mUED frameworks", J. D. Zornoza, Proceedings of VLVnT Workshop, Erlangen, 2011, arXiv:1204.5290 [astro-ph]

[13] "Challenges of Profile Likelihood Evaluation in Multi-Dimensional SUSYScans", arXiv:arXiv:1101.3296

[14] G. Bertone et al., "Global fits of the Minimal Universal Extra Dimensions scenario", Phys.Rev.D 83 036008 (2011) 


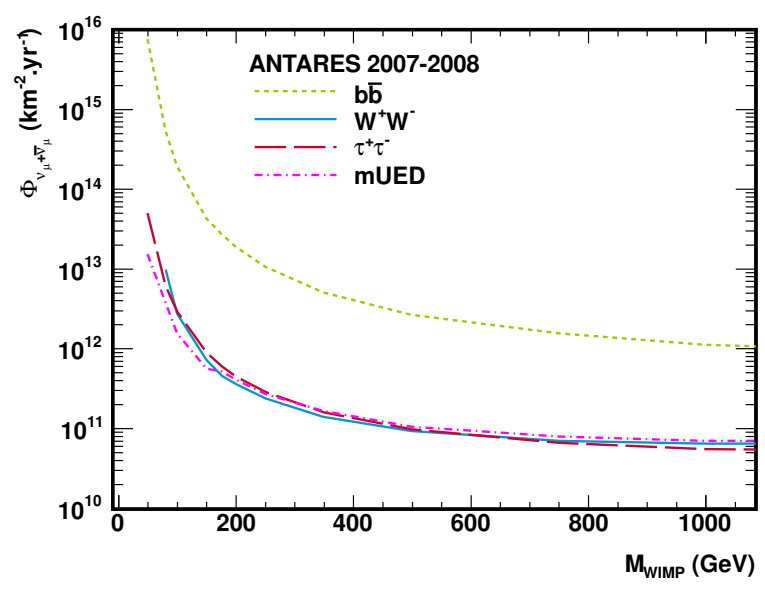

Figure 2: Sensitivity in the neutrino flux as a function of the WIMP mass. (Preliminary).
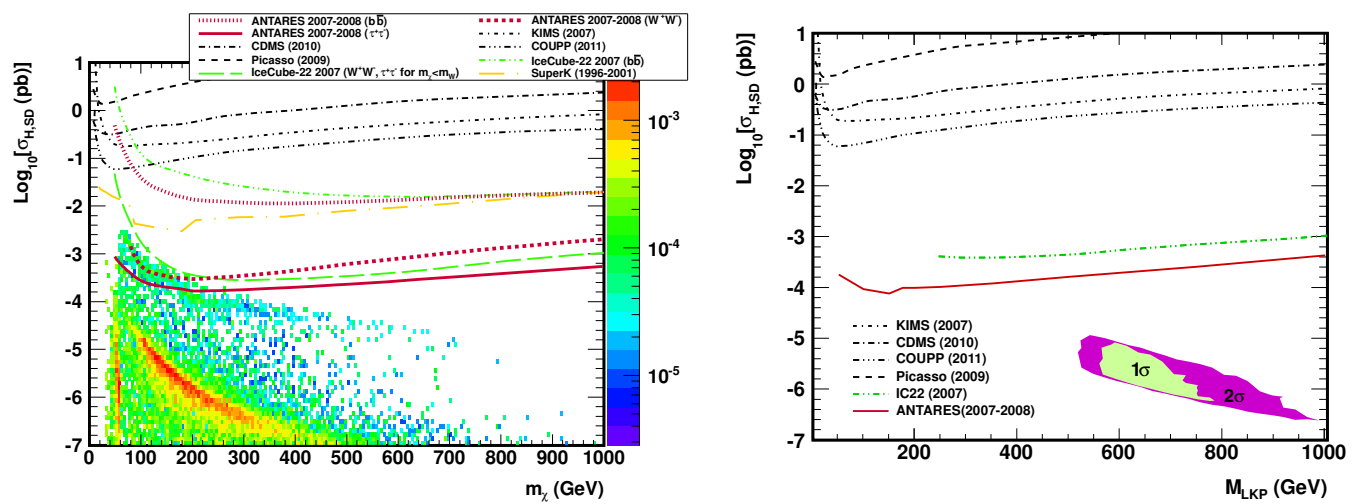

Figure 3: Experimental limits in the spin-dependent cross-section $\bar{\sigma}_{H, S D}$ compared to the theoretical parameter space allowed by the experimental constraints. The left plot corresponds to the CMSSM model. The right plot corresponds to the mUED model, where the light and dark areas correspond to the one and two sigma exclusion regions, respectively. (Preliminary). 